BMJ Open

Sport \&

Exercise

Medicine

\title{
Video analysis of concussion injury mechanism in under-18 rugby
}

\author{
Sharief Hendricks, ${ }^{1}$ Sam O'Connor, ${ }^{1}$ Michael Lambert, ${ }^{1}$ James C Brown, ${ }^{1}$ \\ Nicholas Burger, ${ }^{1}$ Sarah Mc Fie, ${ }^{1}$ Clint Readhead, ${ }^{1,2}$ Wayne Viljoen ${ }^{1,2}$
}

To cite: Hendricks S, O'Connor S, Lambert M, et al. Video analysis of concussion injury mechanism in under-18 rugby. BMJ Open Sport Exerc Med 2016;2:e000053. doi:10.1136/bmjsem-2015000053

- Prepublication history for this paper is available online. To view these files please visit the journal online (http://dx.doi.org/10.1136/ bmjsem-2015-000053).

Accepted 11 September 2015

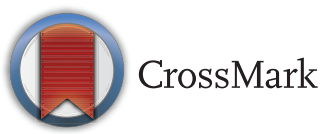

${ }^{1}$ Division of Exercise Science and Sports Medicine, Department of Human Biology, Faculty of Health Sciences, University of Cape Town, Cape Town,

South Africa

${ }^{2}$ South African Rugby Union (SARU), Cape Town,

South Africa

Correspondence to Dr Sharief Hendricks; sharief.hendricks01@gmail. com

\section{ABSTRACT}

Background: Understanding the mechanism of injury is necessary for the development of effective injury prevention strategies. Video analysis of injuries provides valuable information on the playing situation and athlete-movement patterns, which can be used to formulate these strategies. Therefore, we conducted a video analysis of the mechanism of concussion injury in junior-level rugby union and compared it with a representative and matched non-injury sample.

Methods: Injury reports for 18 concussion events were collected from the 2011 to 2013 under-18 Craven Week tournaments. Also, video footage was recorded for all 3 years. On the basis of the injury events, a representative 'control' sample of matched non-injury events in the same players was identified. The video footage, which had been recorded at each tournament, was then retrospectively analysed and coded. 10 injury events ( 5 tackle, 4 ruck, 1 aerial collision) and 83 noninjury events were analysed.

Results: All concussions were a result of contact with an opponent and $60 \%$ of players were unaware of the impending contact. For the measurement of head position on contact, $43 \%$ had a 'down' position, $29 \%$ the 'up and forward' and 29\% the 'away' position $(n=7)$. The speed of the injured tackler was observed as 'slow' in $60 \%$ of injurious tackles $(n=5)$. In 3 of the 4 rucks in which injury occurred $(75 \%)$, the concussed player was acting defensively either in the capacity of 'support' ( $n=2)$ or as the 'jackal' ( $n=1)$.

Conclusions: Training interventions aimed at improving peripheral vision, strengthening of the cervical muscles, targeted conditioning programmes to reduce the effects of fatigue, and emphasising safe and effective playing techniques have the potential to reduce the risk of sustaining a concussion injury.

\section{INTRODUCTION}

A concussion is defined as "a complex pathophysiological process affecting the brain, induced by biomechanical forces". ${ }^{1}$ On the basis of a meta-analysis of 29 papers, Gardner $e t a l^{2}$ reported an overall incidence of match play concussion in men's rugby union of 4.7 concussions per 1000 player match hours (range 0.2-17.1). Considering the level of play, elite players have a rate of 0.40 concussions per 1000 player match

\section{Practical implications}

Better understanding of concussion injury mechanisms in youth rugby union.

- Injury prevention programmes may offer targeted training interventions to prevent concussion in youth rugby.

- A video analysis method to describe and compare injury mechanisms of concussion to a representative non-injury sample.

hours (0.3-7.8), schoolboys 0.6 per 1000 player match hours (0.2-10.6), and community-level or subelite-level players 2.1 concussions per 1000 player match hours $(1.2-6.9){ }^{2}$

Epidemiological studies serve to identify the extent of the problem and, in doing so, satisfy the first step in van Mechelen's 'sequence of prevention' model and the Translating Research into Injury Prevention Practice (TRIPP) model. ${ }^{3}{ }^{4}$ In both injury prevention models, the second step is to establish the aetiology and the mechanisms of injury. A weakness of many sports injury studies is that the injury mechanisms have been insufficiently described to identify suitable injury prevention strategies. ${ }^{5}$ Furthermore, a multifactorial approach is required to account for all the factors involved, that is, the inciting event and also the global and local injury mechanisms. ${ }^{56}$

A systematic video analysis of injuries provides valuable information on the playing situation and athlete movement patterns, which can be used to formulate injury prevention strategies. ${ }^{7}$ Video analysis has been utilised in rugby union to study injury mechanisms in tackle contact situations ${ }^{8-10}$ and specifically for concussion in sports such as American football ${ }^{11}$ and ice hockey. ${ }^{12-14}$ In the Canadian NHL, for example, $3 \frac{1}{2} 2$ years of video records and all cases of medically diagnosed concussions were analysed using a standardised framework for coding the mechanisms of concussions. ${ }^{12-14}$ When 
studying injury using video analysis, the majority of studies are limited to studying the injury event only. The more favourable approach would be to understand the athlete's injury event in comparison with a representative 'control' sample of non-injury events. Using this approach, one would be able to identify differences or deficiencies in the injury event compared with the noninjury event. Moreover, in a technically demanding sport such as rugby, where technical proficiency is a risk factor for injury, ${ }^{15} 16$ this approach will allow for the study of technical discrepancies between the injury and representative non-injury sample.

The implementation of an effective injury prevention strategy relies on a clarification of the extent of the injury problem, and the identification of the injury mechanism. Given the breadth of epidemiological studies on concussion in rugby, the studies identifying the mechanism of injury are lacking. Therefore, we conducted a video analysis of the mechanism of concussion injury in junior level rugby union and compared it with a representative and matched noninjury sample.

\section{METHODS}

\section{Overview}

Injury surveillance was completed as previously described between 2011 and 2013 at the annual South African Rugby Union (SARU) Coca-Cola Youth Week tournaments. ${ }^{17}$ Focusing on the under-18 Craven Week tournaments only, data were obtained from the SARU injury database that is part of an ongoing injury surveillance project coordinated in conjunction with the BokSmart National Rugby Safety Programme. ${ }^{18}$ Video footage containing the concussion injury events at the under-18 Craven Week tournament was also accessed for analysis from the SARU video database. Over the 3 years, 18 concussions were recorded. However, only 10 were available for analysis due to the poor quality of the video footage. On the basis of the 10 injury events ( 5 tackle events- 4 tackler injured and 1 ball-carrier injured; 4 ruck and 1 aerial collision), 83 non-injury events were identified (19 tackle, 61 ruck and 3 aerial collisions). The concussion event was first analysed in a similar fashion to that by Hutchison et al, ${ }^{13}$ using descriptors specific to concussion. Thereafter, both the injury and non-injury events were analysed using coding variables for each phase of play, and for general playing situational variables as outlined by to Hendricks et al. ${ }^{19} 20$ The authors were granted access to these databases by SARU and the UCT Human Research Ethics Committee (injury database HREC Ref: 438/2011; video database HREC Ref: R042/2013).

\section{Injury data collection}

The under-18 Craven Week tournament is an annual fixture aimed at showcasing the country's top schoolboy rugby players. Often a precursor to provincial and national selection, these tournaments are highly contended and thought to be associated with a high injury incidence. ${ }^{15}$ Details about each injury sustained during the tournaments were recorded on an injury collection form that was designed on the basis of the Consensus Statement for injury surveillance. ${ }^{21}$ Information collected included the player's team, body height, body weight, age, whether the player had medical insurance or not, and the presence or absence of protective gear during the inciting event. Written informed consent allowing for the analysis of recorded information was provided prior to the tournament by all players and by the player's parent or legal guardian in the event of the player being younger than 18 years of age. In addition, assent was required by the injured player or by the player's parent or legal guardian at the time of recording the injury. If a player, by virtue of the nature of their injury on the day, was unable to give written assent, verbal assent was acquired after an explanation of the nature of the study was given.

Data were obtained from the annual SARU injury surveillance project that forms part of the BokSmart National Rugby Safety Programme. ${ }^{18}$ Video footage containing the concussion events at the under-18 Craven Week tournament was also accessed for analysis from the SARU video database.

\section{Concussion definition}

Before every tournament, all tournament doctors were informed of the most recent concussion protocols and consensus documents to evaluate and manage concussion. World Rugby (then called IRB) Regulation 10 (http://www.worldrugby.org) and SARU Concussion management protocols (http://www.boksmart.com) were initially used in 2011 and 2012; these were based on the previous 2008 Concussion Consensus Statement. ${ }^{22}$ From 2013 onwards, the updated 2012 Concussion Consensus Statement, ${ }^{1}$ World Rugby Regulation 10 and SARU Concussion protocols were used. These were circulated to the tournament doctors and discussed with them.

\section{Video analysis}

Video footage was analysed using Sports Code elite V.6.5.1, using an Apple iMac (Apple, USA) positioned at eye level. The analysis software allows control over the time lapse during each movement, and the recording and saving of each coded instance into a database. During the analysis, the analyst is at liberty to pause, rewind and watch the footage in slow motion. The highest frequency the analyst was able to slow down the motion of the footage was $25 \mathrm{~Hz}$ (25 frames per second).

Each concussion was analysed using a list of concussion descriptors that were specific to describing the injury mechanism of concussion but non-specific to the phase of play (tables 1 and 2). For example, acceleration of the head, head position precontact and whether the 
Table 1 Definitions for Concussion descriptors

\begin{tabular}{|c|c|}
\hline Operational variable & Definitions \\
\hline \multicolumn{2}{|c|}{ Scenario-identifies the context that precipitated the eventual injury ${ }^{12}$} \\
\hline With teammate & Injury occurs as a result of actions/involvement of teammates \\
\hline With opponent & Injury occurs because of actions/involvement with members of opposing team \\
\hline Fall/trip & Independent of interference from other players, player loses footing and trips/falls \\
\hline Other & Any situation not described by above scenarios \\
\hline Inconclusive & Unable to definitively identify scenario/result of combination of scenarios \\
\hline \multicolumn{2}{|l|}{ Player position } \\
\hline Hooker & Identified by number on playing jersey or on injury report \\
\hline Prop & Identified by number on playing jersey or on injury report \\
\hline Second row & Identified by number on playing jersey or on injury report \\
\hline Flanker & Identified by number on playing jersey or on injury report \\
\hline Number eight & Identified by number on playing jersey or on injury report \\
\hline Scrum Half & Identified by number on playing jersey or on injury report \\
\hline Fly-half & Identified by number on playing jersey or on injury report \\
\hline Centre & Identified by number on playing jersey or on injury report \\
\hline Wing & Identified by number on playing jersey or on injury report \\
\hline Back & Identified by number on playing jersey or on injury report \\
\hline \multicolumn{2}{|c|}{ Body region: concussed player-area of concussive contact $^{12}$} \\
\hline Head/face & Initial contact to area of head/face \\
\hline Neck & Initial contact to neck, that is, area between shoulders and head \\
\hline Shoulders/arms & Initial contact to shoulders/arms \\
\hline Torso & Initial contact to torso, that is, body NOT including head and limbs \\
\hline Hips and below & Initial contact to hip area or below, that is, hip to ankle \\
\hline Inconclusive & Unable to definitively identify body region/combination of regions \\
\hline \multicolumn{2}{|c|}{ Object or body region of other player-anatomical region or object concussed player makes concussive hit onto } \\
\hline Head & Concussion contact to area of head/face \\
\hline Neck & Concussion contact to neck, that is, area between shoulders and head \\
\hline Shoulders/arms & Concussion contact to shoulders/arms \\
\hline Torso & Concussion contact to torso, that is, body NOT including head and limbs \\
\hline Hips and below & Concussion contact to hip area or below, that is, hip to ankle \\
\hline With ground & Concussion contact occurs onto ground \\
\hline With pole & Concussion contact occurs into pole \\
\hline Inconclusive & Unable to definitively identify body region/combination of regions \\
\hline \multicolumn{2}{|c|}{ Body location-refers to anatomical aspect of the region struck (eg, if contact is to forehead=anterior) ${ }^{12}$} \\
\hline Anterior & Front \\
\hline Posterior & Back \\
\hline Lateral & Side \\
\hline Inconclusive & Unable to definitively report \\
\hline \multicolumn{2}{|c|}{ Acceleration direction of head-identifies biomechanical plane(s) of concussed player's head motion ${ }^{12}$} \\
\hline Sagittal & Forward-backward movements \\
\hline Coronal & Side-to-side movements \\
\hline Transverse & Rotational or twisting movements \\
\hline Multiplane & Movements incorporating more than one plane \\
\hline Inconclusive & Unable to definitively identify plane \\
\hline \multicolumn{2}{|c|}{ Head position pre-contact: concussed } \\
\hline Up and forward & Toward object/contact player \\
\hline Away & Away from object/contact player \\
\hline Down & Towards the ground \\
\hline In motion & Player's head was moving \\
\hline \multicolumn{2}{|c|}{ Head position on contact: concussed player } \\
\hline Up and forward & Towards object/contact player \\
\hline Away & Away from object/contact player \\
\hline Down & Towards the ground \\
\hline In motion & Player's head was moving \\
\hline
\end{tabular}

contact was an anticipated hit. The list was largely adapted from the 'heads-up checklist (HUC)', a standardised observational tool designed to describe situational factors and injury mechanisms related to concussion in ice hockey. ${ }^{12}$ Subsequent to the specific analyses of the mechanism of concussion injury, descriptors for the 
Table 2 Definitions for Concussion descriptors

\begin{tabular}{|c|c|}
\hline Operational variable & Definitions \\
\hline \multicolumn{2}{|c|}{ Speed at which player is moving prior to injurious event ${ }^{19} 20$} \\
\hline Stationary & No visible foot movement \\
\hline Slow & Walking \\
\hline Moderate & Jogging (non-purposeful slow running with low knee lift) \\
\hline Fast & Running/sprinting (purposeful running with maximal effort, high knee lift \\
\hline \multicolumn{2}{|c|}{ Speed at which other involved player(s) are moving prior to injurious event ${ }^{19} 20$} \\
\hline Stationary & No visible foot movement \\
\hline Slow & Walking \\
\hline Moderate & Jogging (non-purposeful slow running with low knee lift) \\
\hline Fast & Running/sprinting (purposeful running with maximal effort, high knee lift \\
\hline \multicolumn{2}{|c|}{ Protective gear: concussed player ${ }^{12}$} \\
\hline Scrum cap & Concussed player was wearing a scrum cap \\
\hline Mouthguard & Concussed player was wearing a mouthguard \\
\hline Both & Concussed player was wearing both a scrum cap and a mouthguard \\
\hline None & Player was wearing neither a scrum cap nor a mouthguard \\
\hline \multicolumn{2}{|c|}{ Anticipated hit-whether the player was aware of impending contact situation ${ }^{12}$} \\
\hline Yes & Concussed player was aware of/attuned to impending contact \\
\hline No & Concussed player was unaware of/oblivious to impending contact \\
\hline \multicolumn{2}{|c|}{ On-field medical attention ${ }^{12}$} \\
\hline Yes & Player received immediate on-field medical attention \\
\hline No & Player did not receive immediate on-field medical attention \\
\hline \multicolumn{2}{|l|}{ Game status } \\
\hline First day/stage game & Game is a qualifier taking place on a day other than the final day \\
\hline Final day game & Game is on the final day of the tournament \\
\hline \multicolumn{2}{|c|}{ Score at time of injury—from perspective of concussed player's team } \\
\hline Winning & Concussed player's team is winning the game \\
\hline Losing & Concussed player's team is losing the game \\
\hline Tied game & The game is tied at the time of injury \\
\hline \multicolumn{2}{|c|}{ Time played in current game, minute ${ }^{12}$} \\
\hline $0-20$ & Player has been on the field playing for between 0 and $20 \mathrm{~min}$ \\
\hline 20-40 & Player has been on the field playing for between 20 and $40 \mathrm{~min}$ \\
\hline $40-60$ & Player has been on the field playing for between 40 and $60 \mathrm{~min}$ \\
\hline $60-80$ & Player has been on the field playing for between 60 and $80 \mathrm{~min}$ \\
\hline
\end{tabular}

phase of play (ie, tackle, ruck or aerial collision) in which the concussion occurred were analysed. Tackle descriptors were analysed in similar manner to Hendricks et al. ${ }^{19}{ }^{20}$ Video analysis research for the ruck and aerial collision is limited, and therefore descriptors for the ruck and aerial collision were based on coaching resources and consultations with rugby coaches, sports scientists and rugby administrators. Depending on the phase of play in which the injury occurred (eg, tackle, ruck or aerial collision), all the player's previous noninjury situations in the same phase of play, within the same match and previous matches were identified and analysed as controls. Match situational variables were analysed for all injury and non-injury events.

Statistical analyses was performed by STATA V.12. Given the small sample size, descriptive statistics are reported. Frequency percentage tables were generated for each descriptor under injury and non-injury events. Concussion incidence rate was based on the player match-exposure and the number of concussion events and reported per 1000 player match-hours with corresponding $95 \%$ CIs.

\section{Reliability}

Three players were randomly selected using a random number generator (http://www.random.org/). Each player's injury and non-injury events were coded on two separate occasions, separated by at least 1 week. The $\kappa$ statistics were used to test the intrareliability of the coder for each of the three players. For concussion variables $\kappa=0.92$, for match situation $\kappa=0.98$ and for ruck $\kappa=0.96$. For the tackle, precontact $\kappa=0.77$, contact $\kappa=0.74$, postcontact $\kappa=0.91$, and performance outcome $\kappa=1$. The $\kappa$ values between 0.81 and 0.99 represent 'excellent agreement', and values between 0.61 and 0.80 represent 'substantial agreement'.

\section{RESULTS}

\section{Player demographics}

The average $( \pm \mathrm{SD})$ age, height, mass and body mass index of the concussed players was $18.1 \pm 0.4$ years, 183.2 $\pm 7.9 \mathrm{~cm}, 90.2 \pm 10.1 \mathrm{~kg}$ and $26.9 \pm 2.6 \mathrm{~kg} / \mathrm{m}^{2}$, respectively. For the 10 players analysed in this study, 5 of the concussed players were not wearing protective gear (ie, 
neither a mouthguard nor a scrum cap), 4 players were using a mouthguard and 1 player was using both a mouthguard and a scrum cap.

\section{Performance outcome}

Only one of the injurious tackles was 'completed' $(\mathrm{n}=1$ of the 5), as opposed to $53 \%$ of non-injury tackles ( $n=10$ of the 19). From a tackler perspective, $60 \%$ of concussive tackles were 'unsuccessful ( $\mathrm{n}=3$ of the 5), and 11 of the 19 non-injury tackles were 'successfut' (58\%).

\section{Incidence}

Over the 3 years, 18 concussions were recorded, representing $11 \%$ of all injuries and translating into an incidence rate of 5.8 concussions per 1000 player hours (95\% CI 3.1 to 8.5$)$. There was no significant difference in incidence rate between 2011 and 2012 with a rate of $3.8(95 \%$ CI 0.1 to 7.5$)$ and 3.9 (95\% CI 0.1 to 7.8$)$ concussions per 1000 player hours, respectively. In 2013, the incidence rate increased to 9.52 concussions per 1000 player hours (95\% CI 3.6 to 15.4 ).

\section{Injury mechanism}

A total of 10 concussions were analysed, of which 5 occurred during the tackle ( 4 to the tackler and 1 to the ball-carrier), 4 during the ruck and 1 during an aerial collision (table 3). All 10 concussions analysed occurred after contact with an opponent. In terms of anticipating contact, $60 \%(n=10)$ of the players were unaware of the impending contact. Fifty per cent of the injured players were forwards ( 2 flankers, 2 hookers and 1 lock, respectively) and $50 \%$ were backline players (2 fullbacks, 2 centres and 1 wing, respectively). In $50 \%(n=10)$ of the cases, the injured player's team was losing, 20\% drawing and $30 \%$ winning. On-field medical attention was received immediately after the injury event by 9 of the 10 players. One player was attended to during half time and subsequently removed from the game.

The 'up and forward' head position represented $40 \%$ $(n=10)$ of all precontact head positions while $30 \%$ of players assumed a 'down' head position and 20\% an 'away' position (1 precontact head position was unidentifiable). In head position on contact, $30 \%$ of players were observed displaying a 'down' position, $20 \%$ the 'up and forward' position and $20 \%$ the 'away' position (3 head position on contact events were unidentifiable, $\mathrm{n}=10$ ). Contact to the 'head/face' of the concussed player was noted in all injury events $(n=10)$. In contrast, the body region of the other player most commonly contacted in concussion events was 'hips and below' $(50 \%, \mathrm{n}=10)$.

Seventy per cent of concussions occurred as a result of contact to the 'anterior' side of the head, with $20 \%$ as a result of contact to the 'posterior' side of the head ( 1 body location unidentifiable, $n=10$ ). Acceleration of the head was found to occur within the 'sagittal' plane $50 \%$ of the time. Only $20 \%$ of the concussed players were moving at a 'fast' speed preceding the concussion. The majority $(70 \%, \mathrm{n}=10)$ of injured players were either
Table 3 Frequency and percentage of descriptors for concussive event

\begin{tabular}{|c|c|c|}
\hline & $\mathbf{n}$ & $\%$ \\
\hline \multicolumn{3}{|l|}{ Scenario } \\
\hline With teammate & 0 & 0 \\
\hline With opponent & 10 & 100 \\
\hline Fall/trip & 0 & 0 \\
\hline Other & 0 & 0 \\
\hline Inconclusive & 0 & 0 \\
\hline \multicolumn{3}{|l|}{ Player position } \\
\hline Back & 2 & 20 \\
\hline Centre & 2 & 20 \\
\hline Flanker & 2 & 20 \\
\hline Hooker & 2 & 20 \\
\hline Second row & 1 & 10 \\
\hline Wing & 1 & 10 \\
\hline Prop & 0 & 0 \\
\hline Number eight & 0 & 0 \\
\hline Scrum half & 0 & 0 \\
\hline Fly-half & 0 & 0 \\
\hline \multicolumn{3}{|c|}{ Head position precontact: concussed player } \\
\hline Up and forward & 4 & 40 \\
\hline Down & 3 & 30 \\
\hline Away & 2 & 20 \\
\hline In motion & 0 & 0 \\
\hline \multicolumn{3}{|c|}{ Head position on contact } \\
\hline Up and forward & 2 & 20 \\
\hline Down & 3 & 30 \\
\hline Away & 2 & 20 \\
\hline In motion & 0 & 0 \\
\hline \multicolumn{3}{|c|}{ Body region: concussed player } \\
\hline Head/face & 10 & 100 \\
\hline Neck & 0 & 0 \\
\hline Shoulder/arms & 0 & 0 \\
\hline Torso & 0 & 0 \\
\hline Hips and below & 0 & 0 \\
\hline
\end{tabular}

Body region or object with which concussive contact was made

Head/face

Neck

Shoulder/arms

Torso

Hips and below

With ground

0

0

1

0

5

2

Body location

Anterior

Posterior

Lateral

Acceleration of head

Coronal

Sagittal

Transverse

Multiplane

Speed of concussed player

Stationary

Slow

Moderate

Fast

Speed of other player

Stationary 
Table 3 Continued

\begin{tabular}{|c|c|c|}
\hline & $\mathbf{n}$ & $\%$ \\
\hline Slow & 0 & 0 \\
\hline Moderate & 6 & 60 \\
\hline Fast & 4 & 40 \\
\hline \multicolumn{3}{|c|}{ Movement speed of body region in contact: injured player } \\
\hline Stationary & 3 & 30 \\
\hline Slow & 1 & 10 \\
\hline Moderate & 2 & 20 \\
\hline Fast & 4 & 40 \\
\hline \multicolumn{3}{|c|}{ Movement speed of body region in contact: other player } \\
\hline Stationary & 0 & 0 \\
\hline Slow & 0 & 0 \\
\hline Moderate & 4 & 40 \\
\hline Fast & 4 & 40 \\
\hline \multicolumn{3}{|l|}{ Protective gear } \\
\hline Scrum cap only & 0 & 0 \\
\hline Mouthguard only & 4 & 40 \\
\hline Both & 1 & 10 \\
\hline None & 5 & 50 \\
\hline \multicolumn{3}{|l|}{ Anticipated hit } \\
\hline No & 6 & 60 \\
\hline Yes & 4 & 40 \\
\hline \multicolumn{3}{|c|}{ On-field medical attention } \\
\hline No & 1 & 10 \\
\hline Yes & 9 & 90 \\
\hline \multicolumn{3}{|c|}{ Score at time of injury } \\
\hline Winning & 3 & 30 \\
\hline Losing & 5 & 50 \\
\hline Tied game & 2 & 20 \\
\hline \multicolumn{3}{|c|}{ Time played in current game, minute } \\
\hline 0-20 & 1 & 10 \\
\hline $20-40$ & 3 & 30 \\
\hline $40-60$ & 3 & 30 \\
\hline $60-80$ & 3 & 30 \\
\hline
\end{tabular}

'stationary' (50\%) or moving at a pace categorised as 'slow' (20\%). In contrast, the 'other' players involved in the inciting event were moving at a 'moderate' $(60 \%$, $\mathrm{n}=10)$ and 'fast' (40\%) pace.

\section{Injury and non-injury playing situations}

Sixty per cent $(n=10)$ of injury events occurred in the 'fourth quarter', whereas non-injury events were more evenly distributed between quarters with $45 \%, 24 \%$, $19 \%$ and $12 \%$ occurring in the first, second, third and fourth quarters, respectively $(n=83)$. Two of the four injurious rucks $(50 \%)$ occurred in the 'defensive in-goal area/try-line to defensive $22 \mathrm{~m}$ ', whereas the majority $(53 \%, n=60)$ of non-injury rucks occurred in the 'defensive half-way line to defensive $22 \mathrm{~m}$ '. All $(\mathrm{n}=4)$ aerial collision situations (injury and non-injury) occurred within the 'ball-carriers $22 \mathrm{~m}$ to the half-way line'. Two injurious tackles occurred within the 'ball-carrier's $22 \mathrm{~m}$ to the half way line' and two were within the 'defensive half-way line to defensive $22 \mathrm{~m}$ ', with the remaining one occurring in the 'defensive in-goal area/try-line to defensive 22 m' $(n=5)$. The majority (42\%) of non-injury tackles occurred in the 'defensive half-way line to defensive $22 \mathrm{~m}^{\prime}(\mathrm{n}=19)$.

\section{Tackle descriptors for injury and non-injury events}

The body position of the tackler in injury events was distributed as follows: 20\% 'upright', 20\% 'medium', $20 \%$ 'low', 20\% 'upright to low', 20\% 'medium to low' ( $\mathrm{n}=5$; table 4). The head position of the tackler in $60 \%(\mathrm{n}=5)$ of injury situations was 'up and forward' (20\% 'down', $20 \%$ 'tracking'). The speed of the tackler was observed as 'slow' in $60 \%(\mathrm{n}=5)$ of injurious tackles. The speed of the tackler in matched non-injury tackles were distributed as follows - 42\% 'slow', 32\% 'moderate' and 26\% 'fast' $(n=19)$. The majority $(80 \%, n=5)$ of ball-carriers were travelling 'fast' in injury situations, and in noninjury tackles $63 \%(\mathrm{n}=19)$ were travelling 'fast' and $37 \%$ 'moderately'. The ball-carrier's visual awareness was 'apparent' in $80 \% \quad(n=5)$ of injurious tackles and $83 \%$ $(\mathrm{n}=19)$ of matched non-injury tackle events. However, in the one event where the injury was sustained by the ballcarrier, visual awareness was 'absent'. In all the matched non-injury events for the ball-carrier $(n=6)$, the ballcarrier's visual awareness was observed as 'apparent'.

The direction of the tackle was 'front' in four of the five injurious tackles, with one being from the 'side'. The body regions of the concussed tacklers who were struck were 'head and neck' in $75 \%$ and 'shoulder/arm' in $25 \%(n=4)$. In all matched non-injury tackle events, the body region of the tackler struck was 'shoulder/arm' $(n=19)$. The head placement of the concussed tackler was observed as 'in-front' in three of the four concussive tackles, with the remaining one being 'beside'. The concussed ball-carrier was contacted at the 'shoulder/arm'. Ball-carrier fend was 'absent' in $80 \%$ of injurious tackles, whereas in non-injury tackles $47 \%$ of ball-carriers fended 'moderately', in $42 \%$ fending was 'absent' and in $11 \%$ 'strong' $(\mathrm{n}=19)$.

Three of four concussed tacklers did not drive their legs after contact. The 'legs' were the most common body region to hit the ground first for the tackler in injury $(80 \%)$ and non-injury $(76 \%)$ tackle events $(n=5$ and $n=17$, respectively). The concussed ball-carrier's first body region on the ground was the 'shoulder/arm'.

\section{Ruck descriptors for injury and non-injury events}

In three of the four injurious rucks (75\%), the concussed player was acting defensively either in the capacity of 'support' $(\mathrm{n}=2)$ or as the 'jackal' $(\mathrm{n}=1$; table 5). The role of non-injured players was distributed as follows: $43 \%$ were 'support attack', 39\% 'support defence', $10 \%$ 'jackal' and $8 \%$ 'ball-carrier' (n=61). In three of the four concussive rucks $(75 \%)$, the attacking team was 'clearing the ruck'. In non-injury rucks, the attack was 'clearing the ruck' $52 \%$ of the time and 'protecting the ball' $48 \%(n=61)$ of the time. The defensive team were 'competing for the ball' in $75 \%$ of injurious rucks. Similarly, in non-injury rucks, the defensive team was 'competing for the ball' on the majority $(70 \%$, 
Table 4 Frequency and percentage of descriptors for concussive event $(\mathrm{C})$ and non-injury (NI) event during precontact and contact

\begin{tabular}{|c|c|c|c|c|c|c|c|c|c|}
\hline Precontact & $\begin{array}{l}C \\
\text { (n) }\end{array}$ & $\begin{array}{l}\text { Per } \\
\text { cent }\end{array}$ & $\begin{array}{l}\mathrm{NI} \\
\text { (n) }\end{array}$ & $\begin{array}{l}\text { Per } \\
\text { cent }\end{array}$ & Contact & $\begin{array}{l}C \\
\text { (n) }\end{array}$ & $\begin{array}{l}\text { Per } \\
\text { cent }\end{array}$ & $\begin{array}{l}\mathrm{NI} \\
(\mathrm{n})\end{array}$ & $\begin{array}{l}\text { Per } \\
\text { cent }\end{array}$ \\
\hline Body position of tackler & & & & & Type of tackle & & & & \\
\hline Upright & 1 & 20 & 3 & 16 & Arm & 1 & 20 & 7 & 37 \\
\hline Medium & 1 & 20 & 5 & 26 & Collision & 1 & 20 & 1 & 5 \\
\hline Low & 1 & 20 & 2 & 11 & Jersey & 0 & 0 & 1 & 5 \\
\hline Upright to medium & 1 & 20 & 2 & 11 & Shoulder (dive) & 0 & 0 & 1 & 5 \\
\hline Upright to low & 0 & 0 & 6 & 32 & Shoulder (opposite leading leg) & 1 & 20 & 4 & 21 \\
\hline Medium to upright & 0 & 0 & 0 & 0 & Shoulder (same leading leg) & 2 & 40 & 5 & 26 \\
\hline Medium to low & 1 & 20 & 1 & 5 & Smother tackle & 0 & 0 & 0 & 0 \\
\hline Low to upright & 0 & 0 & 0 & 0 & Tap tackle & 0 & 0 & 0 & 0 \\
\hline Low to medium & 0 & 0 & 0 & 0 & & & & & \\
\hline Tackler stance & & & & & Direction of Tackle & & & & \\
\hline Flat-footed & 1 & 20 & 1 & 5 & Front & 4 & 80 & 8 & 42 \\
\hline Back-foot & 0 & 0 & 1 & 5 & Side & 1 & 0 & 7 & 37 \\
\hline Split forward & 2 & 40 & 10 & 53 & Oblique & 0 & 0 & 2 & 11 \\
\hline No stance & 2 & 40 & 7 & 37 & Behind & 0 & 20 & 2 & 11 \\
\hline Head position of tackler & & & & & Body region of tackler struck & & & & \\
\hline Down & 1 & 20 & 5 & 26 & Legs & 0 & 0 & 0 & 0 \\
\hline Tracking & 1 & 20 & 7 & 37 & Mid-torso & 0 & 0 & 0 & 0 \\
\hline Up and forward & 3 & 60 & 5 & 26 & Shoulder/arm & 2 & 40 & 19 & 100 \\
\hline Away & 0 & 0 & 0 & 0 & Head and neck & 3 & 60 & 0 & 0 \\
\hline Arm position of tackler & & & & & Body region of ball-carrier struck & & & & \\
\hline Elbows bent with hands raised & 0 & 0 & 1 & 5 & Legs & 1 & 20 & 7 & 37 \\
\hline Hands above shoulders & 3 & 60 & 4 & 21 & Mid-torso & 3 & 60 & 5 & 26 \\
\hline \multirow[t]{2}{*}{ Hands dropped } & 2 & 40 & 13 & 68 & Shoulder/arm & 1 & 20 & 7 & 37 \\
\hline & & & & & Head and neck & 0 & 0 & 0 & 0 \\
\hline Speed of tackler & & & & & Ball-carrier fend & & & & \\
\hline Slow & 3 & 60 & 8 & 42 & Absent & 4 & 80 & 8 & 42 \\
\hline Moderate & 0 & 0 & 6 & 32 & Moderate & 1 & 20 & 9 & 47 \\
\hline Fast & 2 & 40 & 5 & 26 & Strong & 0 & 0 & 2 & 11 \\
\hline Speed of ball-carrier & & & & & Tackle sequence & & & & \\
\hline Slow & 0 & 0 & 0 & 0 & One-on-one & 3 & 60 & 13 & 68 \\
\hline Moderate & 1 & 20 & 7 & 37 & Sequential & 1 & 20 & 5 & 26 \\
\hline \multirow[t]{2}{*}{ Fast } & 4 & 80 & 12 & 63 & Simultaneous & 1 & 20 & 1 & 5 \\
\hline & & & & & Attacking sequential & 0 & 0 & 0 & 0 \\
\hline Direction of movement of tackler & & & & & Impact force & & & & \\
\hline Forwards & 4 & 80 & 11 & 58 & Low & 1 & 20 & 3 & 16 \\
\hline Backwards & 0 & 0 & 1 & 5 & Medium & 2 & 40 & 12 & 63 \\
\hline Lateral & 1 & 20 & 7 & 37 & High & 2 & 40 & 4 & 21 \\
\hline \multicolumn{10}{|c|}{ Interpersonal distance between ball-carrier and tackler } \\
\hline Near & 5 & 100 & 11 & 58 & & & & & \\
\hline Moderate & 0 & 0 & 8 & 42 & & & & & \\
\hline Distant & 0 & 0 & 0 & 0 & & & & & \\
\hline \multicolumn{10}{|l|}{ Direction of movement of ball-carrier } \\
\hline Straight & 2 & 40 & 11 & 58 & & & & & \\
\hline Side-step & 1 & 20 & 1 & 5 & & & & & \\
\hline Arcing run & 1 & 20 & 0 & 0 & & & & & \\
\hline Lateral run & 0 & 0 & 3 & 16 & & & & & \\
\hline Diagonal run & 1 & 20 & 4 & 21 & & & & & \\
\hline \multicolumn{10}{|c|}{ Orientation of tackler in relation to ball-carrier } \\
\hline In-front & 4 & 80 & 9 & 47 & & & & & \\
\hline Side & 1 & 20 & 6 & 32 & & & & & \\
\hline Oblique & 0 & 0 & 3 & 16 & & & & & \\
\hline Behind & 0 & 0 & 1 & 5 & & & & & \\
\hline \multicolumn{10}{|l|}{ Ball-carrier visual awareness } \\
\hline Absent & 1 & 20 & 2 & 11 & & & & & \\
\hline Apparent & 4 & 80 & 16 & 84 & & & & & \\
\hline Unsure & 0 & 0 & 1 & 5 & & & & & \\
\hline
\end{tabular}


Table 4 Continued

\begin{tabular}{|c|c|c|c|c|c|c|c|c|c|}
\hline Precontact & $\begin{array}{l}C \\
\text { (n) }\end{array}$ & $\begin{array}{l}\text { Per } \\
\text { cent }\end{array}$ & $\begin{array}{l}\text { NI } \\
\text { (n) }\end{array}$ & $\begin{array}{l}\text { Per } \\
\text { cent }\end{array}$ & Contact & $\begin{array}{l}C \\
\text { (n) }\end{array}$ & $\begin{array}{l}\text { Per } \\
\text { cent }\end{array}$ & $\begin{array}{l}\text { NI } \\
\text { (n) }\end{array}$ & $\begin{array}{l}\text { Per } \\
\text { cent }\end{array}$ \\
\hline \multicolumn{10}{|c|}{ Attacking support } \\
\hline Distant & 0 & 0 & 1 & 5 & & & & & \\
\hline Near & 3 & 60 & 8 & 42 & & & & & \\
\hline Immediate & 2 & 40 & 10 & 53 & & & & & \\
\hline \multicolumn{10}{|c|}{ Defensive support } \\
\hline Distant & 0 & 0 & 1 & 5 & & & & & \\
\hline Near & 5 & 100 & 6 & 63 & & & & & \\
\hline Immediate & 0 & 0 & 12 & 32 & & & & & \\
\hline
\end{tabular}

$\mathrm{n}=61$ ) of occasions. The attack maintained possession in all concussive rucks and in $90 \%$ of non-injury rucks.

\section{Aerial collision for injury and non-injury events}

In the injurious aerial collision, both players involved were unaware of the impending contact $(n=1$; table 5). With regard to the speed of player(s), both players involved in injurious collision were moving at a pace classified as 'fast' (the injured player was the defensive player). In the matched non-injurious aerial collisions, all the defenders were moving at a pace classified as 'moderate' and the attackers were observed as 'slow' on all the occasions. Impact was 'high' in the injury event versus 'low' in all non-injury events. In the inciting event, the concussed player failed to land on (his) feet in a stable manner, whereas in all non-injury events this was maintained $(n=3)$. The 'attacker was higher than defender' in the majority of injury $(100 \%, \mathrm{n}=1)$ and non-injury $(67 \%, \mathrm{n}=3)$ events. In the inciting event, the concussed player failed to land on (his) feet in a stable manner, whereas in all non-injury events this was maintained $(n=3)$.

\section{DISCUSSION}

Insight into the injury mechanism of concussion in youth rugby

This is the first study to describe the global and local injury mechanisms of concussion and compares it with a representative and matched non-injury sample in youth rugby, which is an important aspect in formulating effective injury prevention strategies. ${ }^{5}{ }^{6}$ Over a 3-year period, 18 concussions were recorded, and only 10 were available for analysis due to the poor quality of the video footage. This small sample of concussion events over 3 years highlights the challenge with conducting a study of this nature. Owing to the small sample size, the study should be considered preliminary, and therefore any conclusive remarks are difficult to make. That said, the study provides insight into the injury mechanism of concussion in youth rugby, where otherwise data would not exist, and the data can be interpreted in the context of the current injury prevention strategies for concussion.

\section{Situational factors associated with concussion}

In describing the situational factors associated with concussion, all injuries occurred as a result of contact 'with opponent'. The ability to tolerate and engage in frequent physical contact with the opponent is a prerequisite for participation in rugby. Therefore, it is not surprising that contact with an opponent is a major feature in the series of events leading to concussion. For the concussive events analysed, the players were unaware of the impending contact. This finding is in line with Garraway et $a l^{23}$ who found that tackle injury was more likely to occur when the player was unaware of opponents' movements. An explanation for this is that a player can sustain far greater forces without injury if the cervical muscles are tensed, which occurs when the athlete anticipates the collision. It is postulated that activating the neck and posterior shoulder muscles reduces the risk of concussion by mobilising the head, thereby decreasing the resultant acceleration of impact to the head. $^{24}$

Coaching players to increase awareness and anticipation of ensuing contact may therefore be a worthwhile preventative measure for concussion. ${ }^{24}$

\section{Match period and concussion}

The majority of concussive events took place in the fourth quarter. This finding is consistent with previous literature, highlighting the role of fatigue in concussion risk. ${ }^{25}$ A reduction in tackle technique proficiency has also been associated with fatigue in rugby league players, increasing the potential for unsafe tackles. ${ }^{27}$ In ice hockey, in-game fatigue was a significant predictor of concussion. $^{26}$ This may warrant the need to coach contact technique during a state of fatigue for safe and effective execution during match play. ${ }^{27}$

\section{Tackle contact techniques and concussion}

The tackle situation contributed $50 \%$ of concussion injuries, which is higher than previously reported. ${ }^{28} 29$ The low sample size of this study, however, needs to be considered. The majority of injurious tackles were classified as 'front-on' with injury occurring to the tackler, a finding consistent with others who found that tackles from the front resulted in more injuries. ${ }^{8-10}$ While the 
Table 5 Frequency and percentage of descriptors for concussive event (C) and non-injury event (NI) during aerial collision and ruck

\begin{tabular}{|c|c|c|c|c|c|c|c|c|c|}
\hline Aerial collision & $\begin{array}{l}C \\
(n)\end{array}$ & $\begin{array}{l}\text { Per } \\
\text { cent }\end{array}$ & $\begin{array}{l}\mathrm{NI} \\
(\mathrm{n})\end{array}$ & $\begin{array}{l}\text { Per } \\
\text { cent }\end{array}$ & Ruck & $\begin{array}{l}C \\
(n)\end{array}$ & $\begin{array}{l}\text { Per } \\
\text { cent }\end{array}$ & $\begin{array}{l}\mathrm{NI} \\
(\mathrm{n})\end{array}$ & $\begin{array}{l}\text { Per } \\
\text { cent }\end{array}$ \\
\hline \multicolumn{5}{|l|}{ Number of players involved } & \multicolumn{5}{|l|}{ Role at ruck: player of interest } \\
\hline 2 & 1 & 100 & 0 & 0 & Ball-carrier & 1 & 25 & 5 & 8 \\
\hline 3 & 0 & 0 & 2 & 67 & Jackal & 1 & 25 & 6 & 10 \\
\hline 4 & 0 & 0 & 0 & 0 & Support attack & 0 & 0 & 26 & 43 \\
\hline 5 & 0 & 0 & 1 & 33 & Support defence & 2 & 50 & 24 & 39 \\
\hline \multicolumn{5}{|l|}{ Number of attackers } & \multicolumn{5}{|l|}{ Number of players on attack } \\
\hline 1 & 1 & 100 & 2 & 67 & $1-2$ & 1 & 25 & 10 & 16 \\
\hline 2 & 0 & 0 & 0 & 0 & $3-5$ & 3 & 75 & 49 & 80 \\
\hline 3 & 0 & 0 & 1 & 33 & $6-8$ & 0 & 0 & 2 & 3 \\
\hline \multicolumn{5}{|l|}{ Number of defenders } & \multicolumn{5}{|l|}{ Number of players on defence } \\
\hline 1 & 1 & 100 & 0 & 0 & 0 & 0 & 0 & 3 & 5 \\
\hline \multirow{2}{*}{2} & 0 & 0 & 3 & 100 & $1-2$ & 2 & 50 & 32 & 52 \\
\hline & & & & & $3-5$ & 2 & 50 & 26 & 43 \\
\hline \multicolumn{5}{|c|}{ Awareness of impending contact: attacking player } & \multicolumn{5}{|l|}{ Activity at ruck: attack } \\
\hline Yes & 0 & 0 & 3 & 100 & Clearing the ruck & 3 & 75 & 32 & 52 \\
\hline No & 1 & 100 & 0 & 0 & Protecting the ball & 1 & 25 & 29 & 48 \\
\hline \multicolumn{5}{|c|}{ Awareness of impending contact: defensive player } & \multicolumn{5}{|l|}{ Activity at the ruck: defence } \\
\hline Yes & 0 & 0 & 3 & 100 & Competing for the ball & 3 & 75 & 43 & 70 \\
\hline \multirow[t]{2}{*}{ No } & 1 & 100 & 0 & 0 & Support & 1 & 25 & 10 & 13 \\
\hline & & & & & No active engagement & 0 & 0 & 8 & 16 \\
\hline \multicolumn{5}{|l|}{ Speed of defensive player } & \multicolumn{5}{|l|}{ Infringement } \\
\hline Slow & 0 & 0 & 0 & 0 & No & 3 & 75 & 50 & 82 \\
\hline Moderate & 0 & 0 & 3 & 100 & Yes & 1 & 25 & 11 & 18 \\
\hline Fast & 1 & 100 & 0 & 0 & & & & & \\
\hline \multicolumn{5}{|l|}{ Speed of attacking player } & \multicolumn{5}{|l|}{ Attacker possession } \\
\hline Slow & 0 & 0 & 3 & 100 & Maintained possession & 4 & 100 & 55 & 90 \\
\hline Moderate & 0 & 0 & 0 & 0 & Lost possession & 0 & 0 & 6 & 10 \\
\hline Fast & 1 & 100 & 0 & 0 & & & & & \\
\hline Impact & & & & & & & & & \\
\hline Low & 0 & 0 & 3 & 100 & & & & & \\
\hline Medium & 0 & 0 & 0 & 0 & & & & & \\
\hline High & 1 & 100 & 0 & 0 & & & & & \\
\hline First body region in contact: attac & blayer & & & & & & & & \\
\hline Head/face & 0 & 0 & 0 & 0 & & & & & \\
\hline Neck & 0 & 0 & 0 & 0 & & & & & \\
\hline Shoulder/arms & 0 & 0 & 1 & 33 & & & & & \\
\hline Torso & 0 & 0 & 1 & 33 & & & & & \\
\hline Hips and below & 1 & 100 & 0 & 0 & & & & & \\
\hline Inconclusive & 0 & 0 & 1 & 33 & & & & & \\
\hline First body region in contact: defe & playe & & & & & & & & \\
\hline Head/face & 1 & 100 & 0 & 0 & & & & & \\
\hline Neck & 0 & 0 & 0 & 0 & & & & & \\
\hline Shoulder/arms & 0 & 0 & 1 & 33 & & & & & \\
\hline Torso & 0 & 0 & 2 & 67 & & & & & \\
\hline Hips and below & 0 & 0 & 0 & 0 & & & & & \\
\hline Inconclusive & 0 & 0 & 0 & 0 & & & & & \\
\hline Body position & & & & & & & & & \\
\hline Controlled & 1 & 100 & 3 & 100 & & & & & \\
\hline Uncontrolled & 0 & 0 & 0 & 0 & & & & & \\
\hline Relative position of attacker/defer & & & & & & & & & \\
\hline Attacker higher than defender & 1 & 100 & 2 & 67 & & & & & \\
\hline Defender higher than attacker & 0 & 0 & 0 & 0 & & & & & \\
\hline Same level & 0 & 0 & 1 & 33 & & & & & \\
\hline Lands on feet/stable & & & & & & & & & \\
\hline Yes & 0 & 0 & 3 & 100 & & & & & \\
\hline No & 1 & 100 & 0 & 0 & & & & & \\
\hline
\end{tabular}


Table 5 Continued

\begin{tabular}{|c|c|c|c|c|c|c|c|c|c|}
\hline Aerial collision & $\begin{array}{l}C \\
\text { (n) }\end{array}$ & $\begin{array}{l}\text { Per } \\
\text { cent }\end{array}$ & $\begin{array}{l}\text { NI } \\
\text { (n) }\end{array}$ & $\begin{array}{l}\text { Per } \\
\text { cent }\end{array}$ & Ruck & $\begin{array}{l}C \\
\text { (n) }\end{array}$ & $\begin{array}{l}\text { Per } \\
\text { cent }\end{array}$ & $\begin{array}{l}\text { NI } \\
(n)\end{array}$ & $\begin{array}{l}\text { Per } \\
\text { cent }\end{array}$ \\
\hline \multicolumn{10}{|c|}{ Who gained possession of the ball } \\
\hline Attacker & 0 & 0 & 0 & 0 & & & & & \\
\hline Defender & 0 & 0 & 1 & 100 & & & & & \\
\hline \multicolumn{10}{|l|}{ Infringement } \\
\hline Yes & 0 & 0 & 0 & 0 & & & & & \\
\hline No & 1 & 100 & 3 & 100 & & & & & \\
\hline
\end{tabular}

nature of the game necessitates a high frequency of this type of tackle, the coaching of safe and effective techniques for all tackle types must be emphasised.

'Head up and facing the ball-carrier before contact', that is, 'up and forward', is specified in the list of instructions for safe and effective contact techniques. ${ }^{16} 19$ In this study, concussed players displayed a precontact position of head 'up and forward'. However, on contact, the majority of players displayed a 'down' or 'away' head position. When a player tucks his chin on his chest into the 'down' position during a tackle, the risk for head or neck injury increases. ${ }^{10}$ This may be a target area for coaching interventions as players need to ensure that the 'up and forward' head position is maintained into contact and not only in preparation for contact.

Concussive impact may occur when a person's body and head are travelling at a particular speed and strike a solid object or when a head at rest is struck by a moving object. $^{30}$ In 7 of the 10 concussive events, the injured player was either stationary or moving at a pace categorized as 'slow'. In particular, most injured tacklers were categorised as either 'stationary' or 'slow', suggesting that difference in speed is a risk factor for concussion. Speed differences on contact during rugby have been noted before as a risk factor for injury in rugby. ${ }^{16} 23$ That said, the speed difference between players on contact, as a risk factor for injury, should not be interpreted in isolation. ${ }^{15} 31$

In the majority of injuries to the tackler, leg drive by the tackler was observed to be 'absent'. BokSmart recommendations on safe tackling techniques emphasise the need for a player to maintain leg drive after contact. ${ }^{16}$ This particular technical point also increases the probability of tackle success. ${ }^{19}$

\section{Ruck contact techniques and concussion}

Rucks contributed the second largest proportion of concussions. In three of the four concussive rucks, the team on attack was 'clearing the ruck' while the defensive team was observed to be 'competing for the ball'. These situations are characterised by players darting or charging into a mass of players, exposing players to potential risk of injury. ${ }^{32}$ Considering the observation that three of the four players injured in the ruck were acting in defence ( 2 as 'support' and 1 as 'the jackal'), it seems that players fulfilling a defensive role in the ruck at the time the attack are clearing are more susceptible to concussive impacts. Additionally, all three of the players were unaware of the impending contact at the time of their injuries. It may therefore be useful to incorporate a coaching emphasis on 'maintaining awareness' for players engaging defensively during a ruck.

\section{Strengths, limitations and implications}

As mentioned earlier, the study is limited by its small sample size, and this ultimately restricted the statistical analyses. That said, the data are important as 3 years of concussion data in youth rugby are reported, and should be seen as setting the foundation for a longitudinal study with more injury and non-injury events. Future concussion research and application may benefit more from a refined video analysis coding system, similar to Hutchison et al $\mathrm{s}^{12}$ checklist in ice hockey.

\section{Summary}

This study represents an attempt to move beyond epidemiological studies by establishing the aetiology and injury mechanism underlying youth concussion and, in doing so, progressing the process of injury prevention. On the basis of the findings, training interventions aimed at improving peripheral vision, targeted conditioning programmes to reduce the effects of fatigue and emphasising safe and effective playing techniques should be structured with the goal of reducing the risk of concussion. While an elimination of concussion in rugby is unlikely, continued research in this area is essential to providing a foundation for the development of effective education, evaluation and prevention strategies.

Twitter Follow Sharief Hendricks at @Sharief_H and Mike Lambert at @MikeLambert 01

Acknowledgements The authors would like to thank the medical staff at all of these tournaments for their invaluable assistance with the injury data collection: all the nurses and paramedic staff at each of the tournaments as well as the tournament doctors: Dr Deon Van Tonder, Dr Malebo Mokotedi, Dr Andrea Burmeister, Dr Patho Cele, Dr Charles Smit, Dr Liz Leuenberger, Dr Ferdi Adams and Dr Viaan Vellerman. They would also like to thank SARU's BokSmart National Rugby Safety Programme and Medical Department for commissioning this injury surveillance project. The authors would also like to express their gratitude to the National Research Foundation Post-Doctoral Fellowship for their support.

Contributors SH was responsible for write-up of the manuscript and study conceptualisation. SO was responsible for data collection. NB and SMF 
assisted with data collection and data analyses. CR and WV set up injury data collection and access to video. JCB collected injury data. ML assisted in all aspects. All the authors edited the manuscript.

Competing interests None declared.

Ethics approval This study was granted ethical approval by the University of Cape Town Health Science Human Research Ethics Committee. UCT Human Research Ethics Committee (injury database HREC Ref: 438/2011; video database HREC Ref: R042/2013).

Provenance and peer review Not commissioned; internally peer reviewed.

Data sharing statement No additional data are available.

Open Access This is an Open Access article distributed in accordance with the Creative Commons Attribution Non Commercial (CC BY-NC 4.0) license which permits others to distribute, remix, adapt, build upon this work noncommercially, and license their derivative works on different terms, provided the original work is properly cited and the use is non-commercial. See: http:// creativecommons.org/licenses/by-nc/4.0/

\section{REFERENCES}

1. McCrory $\mathrm{P}$, Meeuwisse $\mathrm{WH}$, Aubry M, et al. Consensus statement on concussion in sport: the 4th International Conference on Concussion in Sport held in Zurich, November 2012. Br J Sports Med 2013;47:250-8.

2. Gardner AJ, Iverson GL, Williams WH, et al. A systematic review and meta-analysis of concussion in rugby union. Sports Med 2014;44:1717-31.

3. Finch $\mathrm{C}$. A new framework for research leading to sports injury prevention. J Sci Med Sport 2006;9:3-9.

4. van Mechelen W, Hlobil H, Kemper HC. Incidence, severity, aetiology and prevention of sports injuries. A review of concepts. Sports Med 1992;14:82-99.

5. Bahr R. Understanding injury mechanisms: a key component of preventing injuries in sport. Br J Sports Med 2005;39:324-9.

6. Usman J, McIntosh AS, Quarrie K, et al. Shoulder injuries in elite rugby union football matches: epidemiology and mechanisms. J Sci Med Sport 2015;18:529-33.

7. Krosshaug T, Verhagen E. Investigating injury risk factors and mechanisms. In: Verhagen E, van Mechelen W, eds. Sports injury research. New York: Oxford University Press, 2010. Chapter 9 109-21.

8. Fuller CW, Ashton TT, Brooks JHM, et al. Injury risks associated with tackling in rugby union. Br J Sports Med 2010;44:159-67.

9. Quarrie KL, Hopkins WG. Tackle injuries in professional rugby union. Am J Sports Med 2008;36:1705-16.

10. Mclntosh AS, Savage TN, McCrory $P$, et al. Tackle characteristics and injury in a cross section of rugby union football. Med Sci Sports Exerc 2010;42:977-84.

11. Pellman EJ, Viano DC, Tucker AM, et al. Concussion in professional football: location and direction of helmet impacts-Part 2. Neurosurgery 2003;53:1328-40.

12. Hutchison MG, Comper P, Meeuwisse WH. An observational method to code concussions in the National Hockey League (NHL): the heads-up checklist. Br J Sports Med 2014;48:125-9.
13. Hutchison MG, Comper $\mathrm{P}$, Meeuwisse $\mathrm{WH}$, et al. A systematic video analysis of National Hockey League (NHL) concussions, part I: who, when, where and what? Br J Sports Med 2015;49:547-51.

14. Hutchison MG, Comper $\mathrm{P}$, Meeuwisse $\mathrm{WH}$, et al. A systematic video analysis of National Hockey League (NHL) concussions, part II: how concussions occur in the NHL. Br J Sports Med 2015;49: 552-5.

15. Hendricks S, Lambert MI. Theoretical model describing the relationship between the number of tackles in which a player engages, tackle injury risk and tackle performance. J Sports Sci Med 2014;13:715-17.

16. Hendricks S, Lambert M. Tackling in rugby: coaching strategies for effective technique and injury prevention. Int J Sport Sci Coach 2010;5:117-36.

17. Brown J, Verhagen $\mathrm{E}$, Viljoen $\mathrm{W}$, et al. The incidence and severity of injuries at the 2011 South African Rugby Union (SARU) Youth Week tournaments. S Afr J Sport Med 2012;24:1-6.

18. Viljoen W, Patricios J. BokSmart-implementing a National Rugby Safety Programme. Br J Sports Med 2012;46:692-3.

19. Hendricks S, Matthews B, Roode B, et al. Tackler characteristics associated with tackle performance in rugby union. Eur J Sport Sci 2014;14:753-62.

20. Hendricks S, Roode B, Matthews B, et al. Defensive strategies in rugby union. Percept Mot Skill 2013;117:65-87.

21. Fuller CW, Molloy MG, Bagate C, et al. Consensus statement on injury definitions and data collection procedures for studies of injuries in rugby union. Br J Sports Med 2007;41:328-31.

22. McCrory $\mathrm{P}$, Meeuwisse $\mathrm{W}$, Johnston $\mathrm{K}$, et al. Consensus statement on concussion in sport-the 3rd International conference on concussion in sport held in Zurich, November 2008. S Afr J Sports Med 2009;21:36-46.

23. Garraway WM, Lee AJ, Macleod DA, et al. Factors influencing tackle injuries in rugby union football. Br J Sports Med 1999;33:37-41.

24. Hanson E, Stracciolini A, Mannix R, et al. Management and prevention of sport-related concussion. Clin Pediatr 2014;53:1221-230.

25. Hollis SJ, Stevenson MR, McIntosh AS, et al. Incidence, risk, and protective factors of mild traumatic brain injury in a cohort of Australian nonprofessional male rugby players. Am J Sports Med 2009;37:2328-33.

26. Stevens S, Lassonde $M$, de Beaumont $L$, et al. In-game fatigue influences concussions in National Hockey League players. Res Sports Med 2008;16:68-74

27. Gabbett TJ. Influence of fatigue on tackling technique in rugby league players. J Strength Cond Res 2008;22:625-32.

28. Kemp SPT, Hudson Z, Brooks JHM, et al. The epidemiology of head injuries in English professional rugby union. Clin J Sport Med 2008;18:227-34.

29. Fuller CW, Taylor A, Raftery M. Epidemiology of concussion in men's elite Rugby-7s (Sevens World Series) and Rugby-15s (Rugby World Cup, Junior World Championship and Rugby Trophy, Pacific Nations Cup and English Premiership). Br J Sports Med 2015;49:478-83.

30. Bailes JE, Hudson V. Classification of sport-related head trauma: a spectrum of mild to severe injury. J Ath/ Train 2001;36:236.

31. Hendricks S, Karpul D, Lambert M. Momentum and kinetic energy before the tackle in rugby union. J Sports Sci Med 2014;13: 557-63.

32. Quarrie KL, Cantu RC, Chalmers DJ. Rugby union injuries to the cervical spine and spinal cord. Sports Med 2002;32:633-53. 